\title{
Anodal transcranial direct current stimulation (tDCS) boosts dominant brain oscillations
}

Caroline Di Bernardi Luft ${ }^{{ }^{*}}$, loanna Zioga ${ }^{1}$, Joydeep Bhattacharya ${ }^{2}$

\footnotetext{
${ }^{1}$ Queen Mary University of London, School of Biological and Chemical Sciences, E1 4NS, United Kingdom

${ }^{2}$ Goldsmiths, University of London, Department of Psychology, London, SE14 6NW, United Kingdom
}

*corresponding author: c.luft@qmul.ac.uk

Key-words: tDCS, brain oscillations, EEG, connectivity, resting state, allometric scaling. 


\section{To the Editor}

The biological mechanisms behind the observed behavioural effects of transcranial direct current stimulation (tDCS) are still unclear and have prevented further clinical applications. The widely adopted explanation is that anodal tDCS increases the excitability of stimulated areas in a polarised manner, based on early studies with animals[1] and humans[2]. However, this explanation is at odds with neuroimaging findings[3-6]. For instance, anodal tDCS was found to increase baseline alpha power[5] (a marker of cortical inhibition), but also to increase gamma power (a marker of excitation) in response to visual stimuli[5]. Additionally, anodal tDCS was found to increase low-frequency oscillations in the underlying tissue without increasing firing rates[6]. Here we suggest a conciliatory explanation that subtle membrane depolarization, caused by weak direct currents (anodal), could make the synchronized neurons more sensitive or responsive to inputs (either excitatory or inhibitory) rather than excitable. A previous study[7] using computational modelling and in-vitro experiments demonstrated that that the modulation caused by direct currents is amplified by the network dynamics. Since synchronised neuronal network activity is manifested in brain oscillations - synchronised assemblies are brought about by strong common inputs[8], we predicted that anodal tDCS would boost dominant brain rhythms rather than fast brain rhythms. We also predicted an increase in directed connectivity towards the anodal region, i.e. the area would be more receptive to input connections rather than driving other regions.

To test our predictions, we measured brain oscillations and directed phase synchronization before and after three five-minute bouts of anodal $(n=21)$, cathodal $(n=21)$, and sham $(n=$ 18) high-definition tDCS on the right temporal area. We measured dominant brain oscillations using the Better OSCillation detection method[9] which is suitable for nonstationary time series and sensitive to transient changes in brain oscillatory activity. The dominant frequency was defined as the oscillation frequency which had the largest duration in relation to the duration of the recorded (clean) data ( $p$-episode). Phase synchronization 
was estimated by phase slope index[9], which is suitable for finding the direction of information flow between regions and robust against volume conduction.

\section{Results}

Oscillatory activity at the dominant frequency (for each individual) in the stimulation electrode region was analysed using a 3 (stimulation: cathodal, sham, anodal) x 2 (session: before vs. after) mixed ANOVA. We found an increase in dominant oscillatory activity after anodal and a reduction after cathodal compared to sham (session ${ }^{\star}$ stimulation: $F_{(2,57)}=5.11, p=.009$, partial $\left.\eta^{2}=.152\right)$. There were no main effects for session $\left(F_{(1,57)}=0.308, p=.581\right.$, partial $\eta^{2}$ $=.005)$ or stimulation $\left(F_{(2,57)}=0.704, p=.499\right.$, partial $\left.\eta^{2}=.024\right)$, suggesting that the changes in oscillatory activity were dependent on the stimulation condition. Post-hoc comparisons indicated a large effect size for the difference between oscillatory changes following anodal and cathodal (Fig. $1 \mathrm{~B}, t_{(40)}=3.185, p=.003$, Cohen's $d=1.006$, C.I. $(\mathrm{d})=$ 0.962:1.051). A 4 (time periods: before, after 5, 10, and 15 minutes) $\times 3$ (stimulation: cathodal, sham, and anodal) mixed ANOVA revealed an interaction between time periods and stimulation $\left(F_{(6,171)}=6.335, p=.003\right.$, partial $\left.\eta^{2}=.182\right)$; the time course of these changes show that the effects of cathodal decreased quickly after 5 minutes whereas the effects of anodal stimulation took more time to build(Fig. 1A). There were no significant effects of anodal or cathodal tDCS on oscillatory activity over classical frequency bands or on the individual alpha peak frequency (see Supplemental information).

We observed an increase in directed theta phase synchronization, but only towards the stimulated area (i.e. incoming connections). A 2 (session) x 3 (stimulation) mixed ANOVA revealed an increase in incoming connections following anodal tDCS (stimulation*session: $F_{(2,57)}=3.63, p=.033$, partial $\left.\eta^{2}=.113\right)$. This stimulation specific increase in incoming connectivity was higher for anodal stimulation compared to cathodal $\left(t_{(40)}=2.59, p=.013\right.$, Cohen's $d=0.818$, C.I. $(d)=0.602: 1.035)$. Including the time course of these over bouts of 5 minutes revealed a significant interaction between time periods and stimulation $\left(F_{(2,57)}=\right.$ 
4.50, $p=.015$, partial $\eta^{2}=.136$ ), suggesting that the differences between the stimulation conditions were built over time (Fig. 1C). We observed an increase in incoming connections from posterior brain areas to the stimulation area and a reduction from the stimulated areas to the left frontal following anodal tDCS, but no change following cathodal and sham stimulation (Fig.1D).

\section{Discussion and future directions}

We suggest that tDCS boosts dominant brain oscillations by potentially rendering synchronized neurons under anodal tDCS more responsive to inputs. This neuronal account of tDCS could explain a number of seemingly inconsistent tDCS effects on neural responses such as 1) increased alpha oscillations[5]: alpha oscillations are dominant inhibitory inputs; 2) increased motor evoked potentials[2]: MEPs are triggered by a strong input - TMS pulse; 3) increased ERP responses[4, 10]: stimulus processing by is facilitated through time-locked synchronized neurons; 4) large variability in the effects of tDCS on undirected connectivity[3, 11]: effects depend on the connectivity direction; 5) increases in oscillatory responses during tasks[5, 10]: neural responses are facilitated through synchronized activity associated with task demands; 6) increase in low-frequency oscillations in the underlying tissue without increasing firing rates[6]: the low-frequency oscillations were dominant on these monkeys spectrum. In the future, it would be interesting to investigate how these dominant brain oscillations affect the online effects of tDCS.

Considering that networks of neurons are more sensitive to weak currents $(<1 \mathrm{mV} / \mathrm{mm})$ than single neurons[7], we hypothesise that the larger the active network receiving the stimulation, the lower the current needed to affect its neurons. Such economy of scale is ubiquitous in nature, which often shows sub-linear scaling[12] (e.g. 3/4 power law for metabolic rates: larger animals have lower metabolic rates). For instance, if we estimate a $3 / 4$ power law for current sensitivity of neurons (e.g. starting from around $10 \mathrm{mV} / \mathrm{mm}$ ), we postulate that very small currents could affect a larger group of neurons such as those 
generating dominant EEG oscillations. Increased sensitivity scaled to the size of the active network implies that anodal tDCS can facilitate ongoing processes, which can also explain why the effects of the stimulation are critically dependent on brain states. This scaling effect brought about by synchronized activity could explain why currents as low as $0.4 \mathrm{~V} / \mathrm{m}$ (for $2 \mathrm{~mA}$ ), as recently observed during tDCS [13], could modulate brain activity and therefore affect cognitive and affective functions. A key step in the future is to determine the scaling exponent of this sensitivity effect, which will require measuring neural response of exponentially scaled neuronal assemblies (from a single neuron to millions) to varying levels of current (scaled in the same proportion).

We suggest that understanding how synchronized brain activity interacts with weak electrical currents may be a key to explain the large individual differences in the effects of tDCS, statedependency and also to create more efficient tDCS protocols.

\section{Conflicts of interest}

The corresponding author states on behalf of all authors that there are no conflicts of interest.

\section{Acknowledgments}

We would like to thank Oliwia Zabarowska for assisting with data collection.

\section{References}

1. Bindman, L.J., O.C. Lippold, and J.W. Redfearn, Long-lasting changes in the level of the electrical activity of the cerebral cortex produced bypolarizing currents. Nature, 1962. 196: $\mathrm{p}$. 584-5. 
2. Nitsche, M.A. and W. Paulus, Excitability changes induced in the human motor cortex by weak transcranial direct current stimulation. J Physiol, 2000. 527 Pt 3: p. 633-9.

3. Polanía, R., M.A. Nitsche, and W. Paulus, Modulating functional connectivity patterns and topological functional organization of the human brain with transcranial direct current stimulation. Hum Brain Mapp, 2011. 32(8): p. 1236-1249.

4. Reinhart, R.M. and G.F. Woodman, Causal control of medial-frontal cortex governs electrophysiological and behavioral indices of performance monitoring and learning. I Neurosci, 2014. 34(12): p. 4214-27.

5. Wilson, T.W., et al., tDCS Modulates Visual Gamma Oscillations and Basal Alpha Activity in Occipital Cortices: Evidence from MEG. Cereb Cortex, 2017: p. 1-13.

6. Krause, M.R., et al., Transcranial Direct Current Stimulation Facilitates Associative Learning and Alters Functional Connectivity in the Primate Brain. Current Biology, 2017.

7. Reato, D., et al., Low-Intensity Electrical Stimulation Affects Network Dynamics by Modulating Population Rate and Spike Timing. The Journal of Neuroscience, 2010. 30(45): p. 15067-15079.

8. Mirollo, R.E. and S.H. Strogatz, Synchronization of pulse-coupled biological oscillators. SIAM Journal on Applied Mathematics, 1990. 50(6): p. 1645-1662.

9. Whitten, T.A., et al., A better oscillation detection method robustly extracts EEG rhythms across brain state changes: the human alpha rhythm as a test case. Neuroimage, 2011. 54(2): p. 860-74.

10. Reinhart, R.M., et al., Electrical Stimulation of Visual Cortex Can Immediately Improve Spatial Vision. Curr Biol, 2016. 26(14): p. 1867-72.

11. Mancini, M., et al., Assessing cortical synchronization during transcranial direct current stimulation: A graph-theoretical analysis. Neuroimage, 2016. 140: p. 57-65.

12. West, G.B., J.H. Brown, and B.J. Enquist, $A$ general model for the origin of allometric scaling laws in biology. Science, 1997. 276(5309): p. 122-126.

13. Huang, Y., et al., Measurements and models of electric fields in the in vivo human brain during transcranial electric stimulation. Elife, 2017. 6. 
A.

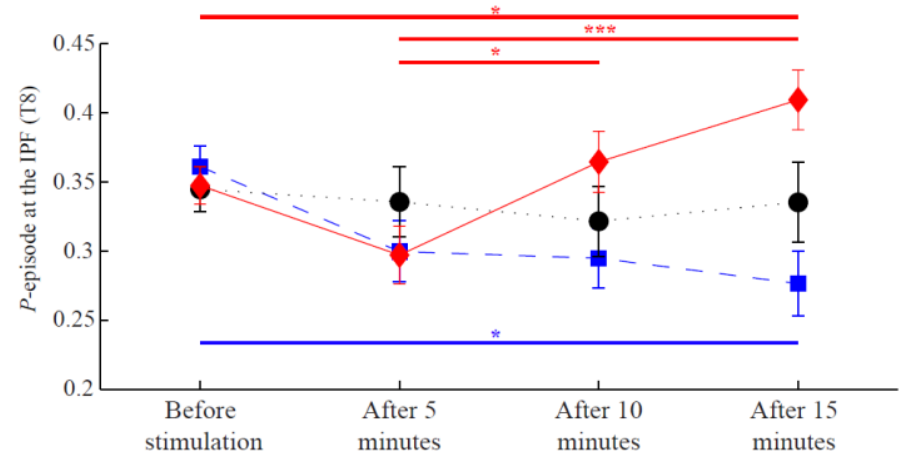

C.

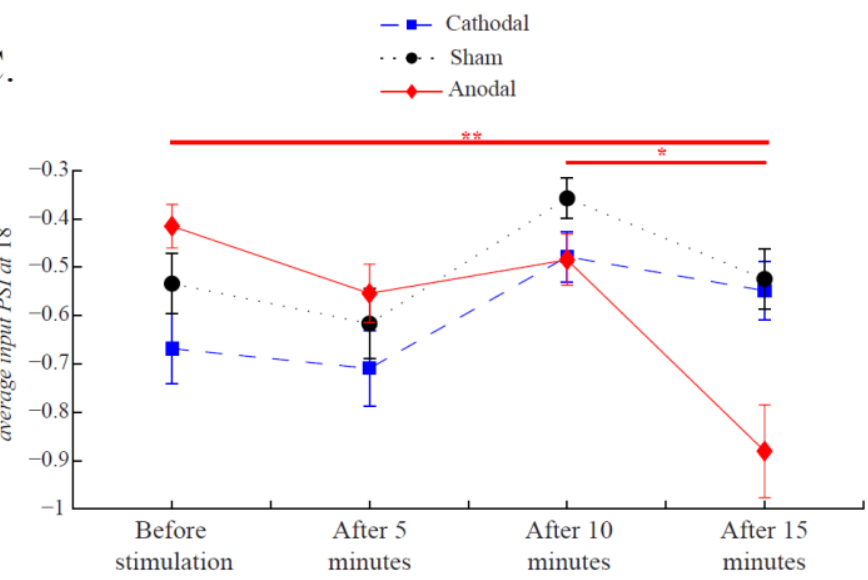

B.

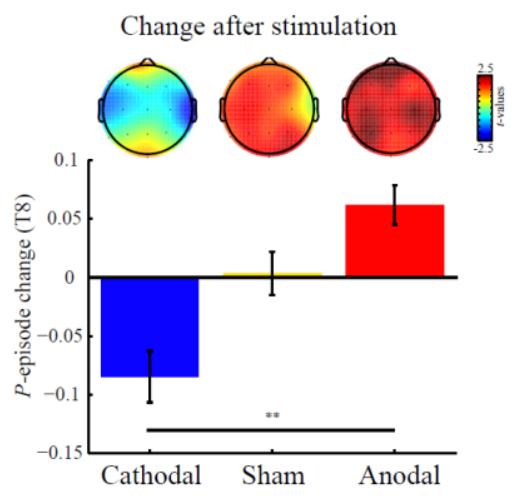

D.

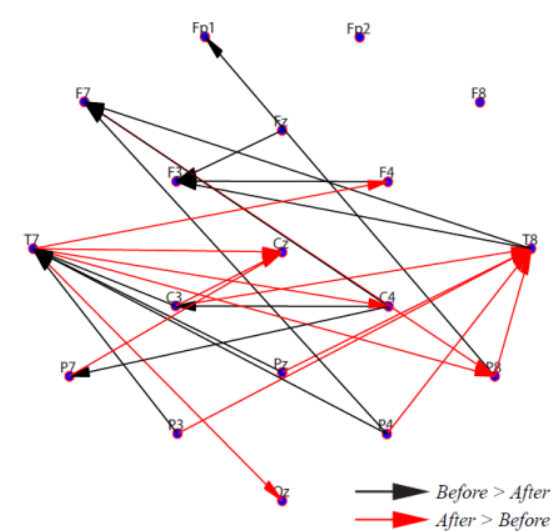

Figure 1. A. Time course of the proportion of the oscillatory activity ( $P$-episode) in the individual peak frequency (IPF) at the right temporal electrode (T8) following cathodal, sham and anodal tDCS. B. Changes in the IPF oscillatory activity (after minus before) at T8 for each stimulation condition and the topography of these contrasts (t-values). C. Time course of the directed connectivity averaged over the input connections (negative PSI) following each stimulation condition. D. Significant connectivity differences between before and after anodal tDCS. Black arrows indicate significant synchronization which was higher in before the stimulation compared to after, and red vice-versa. ${ }^{*} p<.05,{ }^{* *} p<.01,{ }^{* * *} p<.001$. Error bars represent +/- 1 S.E.M. 